\title{
Review
}

\section{Mechanisms of ubiquitin transfer by the anaphase-promoting complex}

\author{
Mary E Matyskiela, Monica C Rodrigo-Brenni and David O Morgan
}

Address: Departments of Physiology and Biochemistry and Biophysics, University of California, San Francisco, CA 94158, USA.

Correspondence: David O Morgan. Email: David.Morgan@ucsf.edu

\begin{abstract}
The anaphase-promoting complex (APC) is a ubiquitin-protein ligase required for the completion of mitosis in all eukaryotes. Recent mechanistic studies reveal how this remarkable enzyme combines specificity in substrate binding with flexibility in ubiquitin transfer, thereby allowing the modification of multiple lysines on the substrate as well as specific lysines on ubiquitin itself.
\end{abstract}

The function of a ubiquitin-protein ligase, as its name implies, is to catalyze the ligation of the small protein ubiquitin to other proteins. Ubiquitin attachment occurs primarily at lysine residues, and in many cases the reaction is repeated at several lysines on the same protein and on ubiquitin itself, resulting in decoration of the target protein with multiple polyubiquitin chains, which send the protein to the proteasome for destruction or alter other aspects of protein function $[1,2]$. Protein ubiquitination is a versatile and effective mechanism for controlling protein behavior, and has been adapted for diverse purposes by many regulatory systems in the eukaryotic cell.

There is enormous complexity in the mechanisms that allow ubiquitin-protein ligases to recognize and repeatedly modify specific substrate proteins. Our purpose here is to illustrate these mechanisms in the case of an unusually large and complex ubiquitin-protein ligase called the anaphase-promoting complex or cyclosome (APC), which promotes the proteasomal destruction of key mitotic regulators in all eukaryotic cells (for reviews, see [3-6]; Figure 1). The APC, like other ubiquitin-protein ligases or E3s, promotes the transfer of ubiquitin to a target protein from another protein called a ubiquitin-conjugating enzyme or E2 (Figure 2a). The APC can therefore be viewed as a platform with specific binding sites for two substrates: the E2-ubiquitin conjugate and the target protein, which are positioned by the APC to allow the $\varepsilon$-amino group of a target lysine to attack the thioester bond that links the carboxyl terminus of ubiquitin to the active-site cysteine of the E2 (Figure 2b).

The APC must bind substrate proteins with high specificity to ensure that only the appropriate targets are destroyed.
In addition, the APC and/or its substrates must be flexible enough to allow the transfer of ubiquitin to multiple lysines on the target protein and to specific lysines on ubiquitin itself (Figure 2a). We will describe the basis for this balance of specificity and flexibility by focusing on two key issues: substrate binding and the catalysis of ubiquitin transfer. These features of APC mechanism are likely to have important implications for ubiquitin-protein ligases in general.

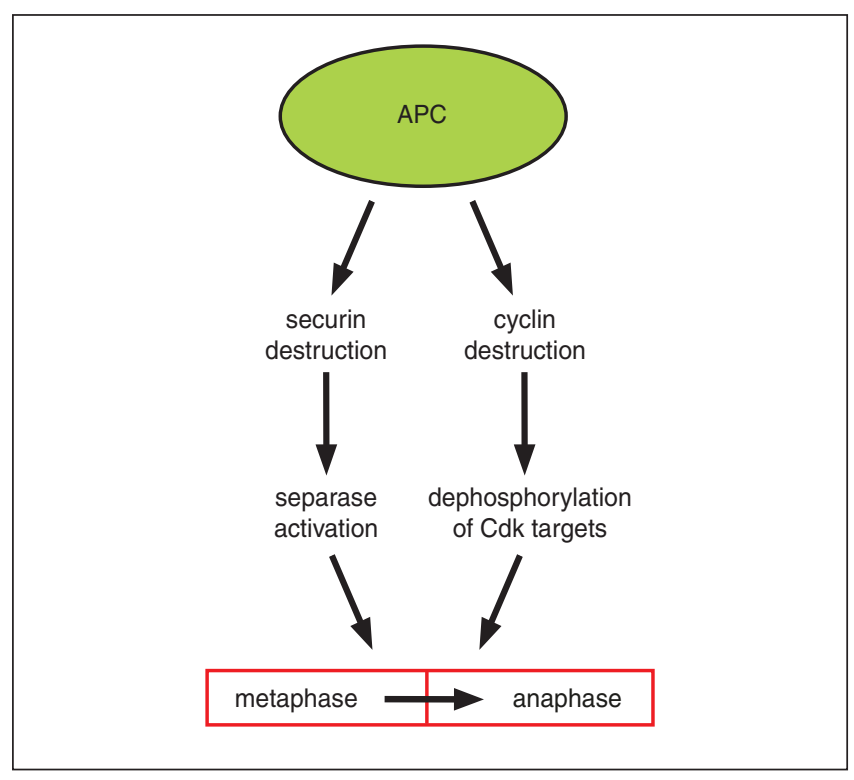

Figure 1

The major biological function of the APC is to initiate chromosome segregation in mitosis. In metaphase, before APC activation, the duplicated chromosomes, or sister chromatids, are aligned at the center of the mitotic spindle. The APC promotes the ubiquitination and proteasomal destruction of securin, thereby unleashing a protease, separase, that cleaves cohesin proteins holding the sister chromatids together. The chromatids separate and are then pulled apart by the mitotic spindle in anaphase. The APC also triggers destruction of cyclins, the activating subunits of the cyclindependent kinases (Cdks). As a result, Cdk activity drops in anaphase. The resulting dephosphorylation of Cdk substrates is required for normal anaphase spindle function and for the completion of mitosis. The APC has numerous additional substrates, not shown here, that contribute to the control of late mitotic events and govern entry into the following $\mathrm{G} 1$. 
(a)

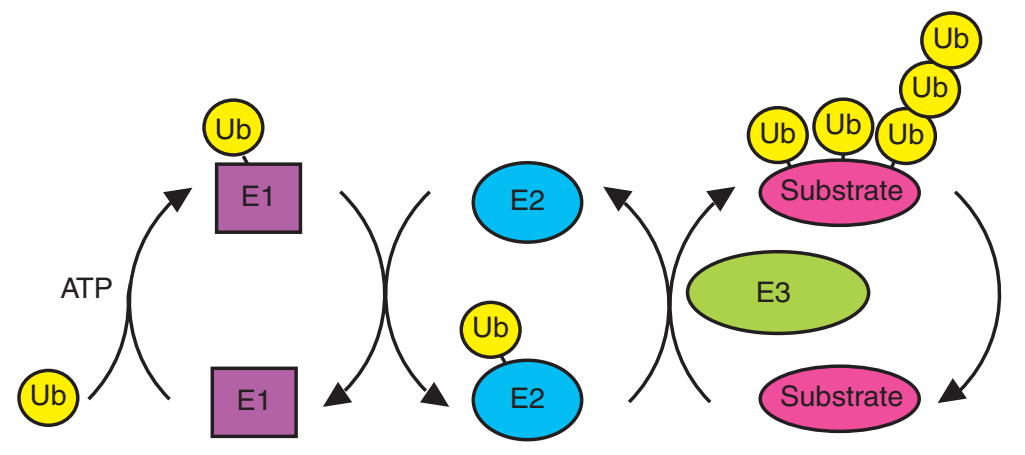

(b)
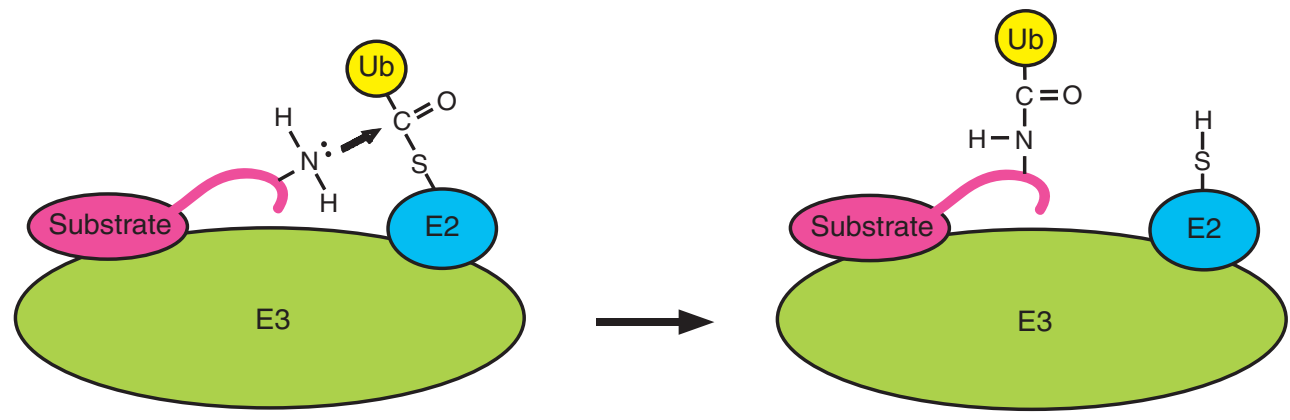

Figure 2

Ubiquitination occurs by a three-enzyme cascade. (a) Ubiquitin (Ub, yellow) is first activated by an E1, or ubiquitin-activating protein (purple square), which couples ATP hydrolysis to the formation of a thioester bond between the active-site cysteine of the E1 and the carboxyl terminus of ubiquitin. The E1 then transfers the activated ubiquitin to the active-site cysteine of an E2, or ubiquitin-conjugating enzyme (blue). Finally, the E3, or ubiquitin-protein ligase (green), facilitates the transfer of the ubiquitin from the E2 to a lysine on the target protein (substrate, magenta). In the case of the APC and many other E3s, this final step is repeated several times with the same substrate, resulting in ubiquitination of multiple lysines. In addition, specific lysines on ubiquitin itself can be modified, resulting in the assembly of polyubiquitin chains. (b) The APC is a member of the RING-domain family of E3s. These proteins facilitate the final step in ubiquitination by positioning the E2-ubiquitin conjugate next to the substrate, allowing the $\varepsilon$-amino group of a lysine on the substrate to nucleophilically attack the E2-ubiquitin thioester bond, resulting in direct transfer of ubiquitin as shown here. Members of the other major family of E3 proteins, called the HECTdomain E3s, employ an indirect, two-step mechanism (not shown here): ubiquitin is first transferred from the E2 to an active-site cysteine in the E3, after which a lysine in the substrate attacks the E3-ubiquitin thioester bond.

\section{Key components of the APC reaction}

Protein ubiquitination by the APC requires the cooperation of four protein components: the APC core, the activator subunit, E2 and substrate (Figure 3). Activators, E2s and substrates all bind reversibly to the APC core with varying affinities, and interact with each other as well. To understand the contribution of each of these components to the ubiquitination reaction, it is helpful to first summarize their basic features.

\section{APC core}

The APC is an approximately $1 \mathrm{MDa}$, tightly associated complex of 11 to 13 subunits that are generally well conserved in eukaryotes (Figure 4; Table 1). The APC is a cullin-RING-type ubiquitin-protein ligase [7], in which the Apc2 and Apc11 subunits contain the cullin and RING domains, respectively. As in other cullin-RING ligases, the RING domain of Apc11 interacts directly with the E2, and the cullin domain of Apc2 binds Apc11 and probably provides an extended scaffold that connects these two subunits to the rest of the enzyme.

The analysis of APC purified from yeast strains lacking individual subunits has led to the identification of APC subcomplexes (Figure 4) [8]. One contains Apc2 and Apc11, as well as a third subunit, Doc1. Doc1 contains a $\beta$-barrel structure known as a Doc domain, which in other proteins is involved in binding to small ligands, and this subunit may contribute to substrate binding, as discussed later. The other APC subcomplex contains three large subunits (Cdc27, Cdc16 and Cdc23 in yeast) that carry ten or more copies of a 34-residue sequence motif called a 


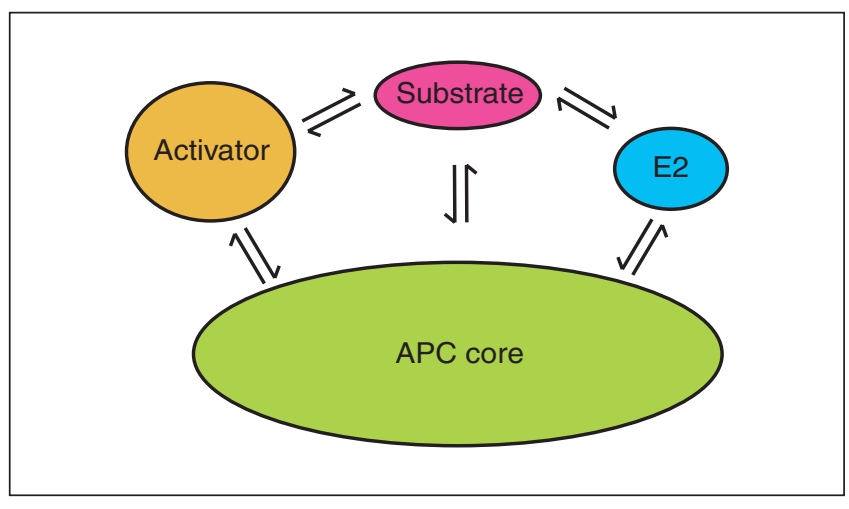

Figure 3

The four major protein components in an APC reaction. Catalysis depends on cooperative interactions among the APC core, activator, substrate and E2.

tetratricopeptide repeat (TPR). These subunits seem to associate sequentially with the APC, such that the association of $\mathrm{Cdc} 27$ depends on $\mathrm{Cdc16}$, and the association of Cdc16 depends on Cdc23 [8]. Stoichiometry calculations suggest that the TPR subunits are present in two copies on the APC $[9,10]$. TPRs generally form protein-binding grooves, and thus the multiple TPR subunits are likely to provide a large array of interaction surfaces on the APC core.

The two APC subcomplexes are held together by the largest APC subunit, Apc1 (Figure 4). Apc4 and Apc5 help connect Apc1 to the base of the TPR subcomplex, Cdc23. The nonessential subunits Cdc26 and Swm1 (not shown in the figure) help stabilize the association of the TPR subunits with the rest of the APC [11,12]. Cdc26 promotes APC integrity by forming a complex with the TPR grooves of Cdc16 [13]. The functions of other APC subunits remain unclear.

Several electron microscopic (EM) analyses have provided a glimpse of the size and shape of the APC [9,10,14-16]. At a resolution of around $30 \AA$, the yeast APC seems to form a triangular particle, and the localization of individual subunits by antibody labeling is roughly consistent with the architecture determined from subcomplex studies $[10,16]$. In the highest-resolution EM structure, the TPR subunits are localized to an 'arc lamp' structure, and Apc2 is found in an adjacent 'platform' region where E2s are likely to bind [16].

\section{Activator}

Despite its large size, the APC core has little activity in the absence of one of its activator proteins, Cdc20 or Cdh1 (a third activator, Ama1, is expressed solely in meiosis and will not be discussed here [17]). Cdc20 associates with the APC in early mitosis, leading to the destruction of targets that control the onset of anaphase. Cdc2o binding to the APC is promoted by phosphorylation of multiple APC subunits [18-23]. Later in mitosis, Cdc20 is replaced by Cdh1, which maintains activity through the following G1. The association of Cdh1 with the APC depends on Cdh1 dephosphorylation $[20,24,25]$.

Activator proteins participate in substrate recognition by the APC. The carboxy-terminal regions of Cdc20 and Cdh1 contain a WD40 domain that is thought to form a propeller-like binding platform that binds APC substrates [26-29]. It is likely that sequence variations in the WD40 domains of Cdc2O and Cdh1 result in different substrate specificities. These differences in specificity provide a mechanism for timing the destruction of different APC targets in mitosis: Cdc20 targets a small number of key substrates for destruction at metaphase, whereas Cdh1 possesses a broader specificity, targeting these proteins and many more in late mitosis and G1 [30,31].

Activators also contain at least two sequence motifs, the Ile-Arg (IR) motif and C-box, that are required for activator binding to the APC core. The IR motif consists of the two residues at the carboxyl terminus of the activator, and the C-box is an eight-residue motif near the amino terminus $[29,32,33]$. Activator binding to the APC is at least partly mediated by the TPR subunits (Figure 4): Cdh1 binds

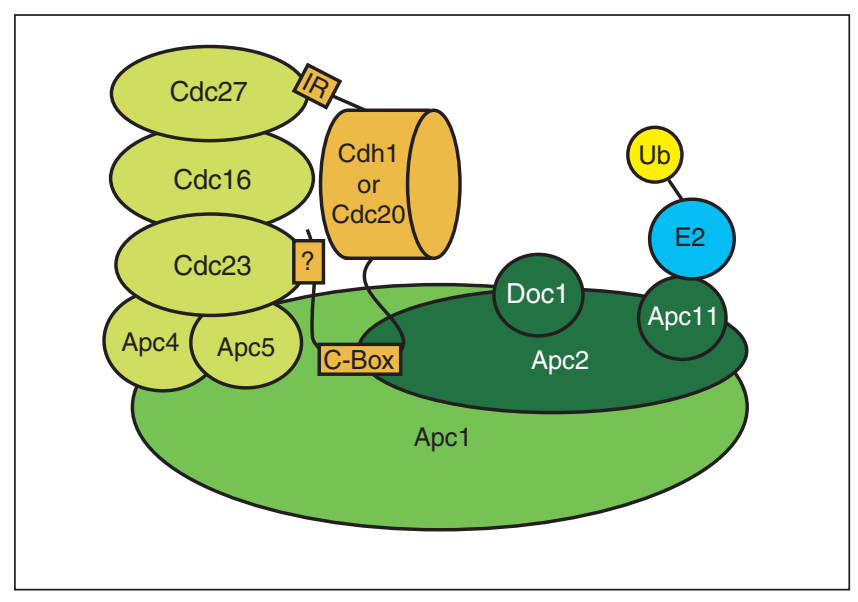

Figure 4

The APC core contains multiple subcomplexes. The budding yeast APC core is an approximately $1 \mathrm{MDa}$ complex of 13 subunits (Table 1), including the nine key subunits shown here. One subcomplex (dark green) contains the cullin subunit Apc2 and the RING-domain protein Apc11, which recruits E2s. Another subcomplex (light green) contains the three TPR-containing subunits, Cdc27, Cdc23 and Cdc16, as well as two subunits, Apc4 and Apc5, that help connect them to the rest of the APC via Apc1. The TPR-containing subunits provide binding sites for the activator (Cdh1 or Cdc20), which contains at least two APC-interaction motifs, the lle-Arg (IR) motif and the C-box, as well as a large WD40 repeat sequence that is likely to form a propeller-like binding site for substrate. 


\begin{tabular}{|c|c|c|c|c|}
\hline S. cerevisiae & S. pombe & Mammals & D. melanogaster & Comments \\
\hline \multicolumn{5}{|l|}{ Core subunits } \\
\hline Apc1 & Cut4 & Apc1/Tsg24 & & \\
\hline Apc2 & Apc2 & Apc2 & & Cullin domain \\
\hline Cdc27 & Nuc2 & Cdc27 & & TPRs \\
\hline Apc4 & Lid1 & Apc4 & & \\
\hline Apc5 & Apc5 & Apc5 & & \\
\hline Cdc16 & Cut9 & Apc6 & & TPRs \\
\hline- & - & Apc7 & & TPRs \\
\hline Cdc23 & Cut23 & Cdc23 & & TPRs \\
\hline Apc9 & - & - & & \\
\hline Doc1 & Apc10 & Apc10 & & Doc domain \\
\hline Apc11 & Apc11 & Apc11 & & RING finger \\
\hline Cdc26 & Hen1 & Cdc26 & & \\
\hline Swm1 & Apc13 & - & & \\
\hline Mnd2 & Apc15 & - & & \\
\hline- & Apc14 & - & & \\
\hline \multicolumn{5}{|l|}{ Activators } \\
\hline Cdc20 & SIp1 & Cdc20/p55 CDC & Fizzy/FZY & WD40 repeats \\
\hline Cdh1/Hct1 & Srw1/Ste9 & Cdh1 & Fizzy-related/FZR & WD40 repeats \\
\hline Ama1 & Mfr1 & & & Meiosis-specific \\
\hline
\end{tabular}

Dashes indicate that no homologous subunit has been identified in that species. Most core subunits are not yet identified in Drosophila melanogaster. Motifs in APC subunits include tetratricopeptide repeats (TPRs) and WD40 repeats, both of which form domains that are typically involved in proteinprotein interactions. Modified from [6].

directly to Cdc27 in vitro [26,32], and specific residues in the protein-interaction grooves formed by the TPRs in Cdc27 and Cdc23 are required for the binding of both Cdh1 and Cdc20 [34]. The activator IR motif binds to the TPRs of $\mathrm{Cdc27}$, and an additional unidentified activator region seems to bind the TPRs of Cdc23 [34]. The C-box binding site remains unknown, but one possibility is the Apc2 subunit, as removal of Apc2 from the APC reduces activator binding [8]. Together, these multiple interactions generate very high-affinity binding of activator to the APC core, and it is likely that the activator remains bound during multiple substrate-binding events [34]. Recent EM analyses suggest that the activator is found between the TPR arc lamp and Apc2, in an ideal position to present substrates to attack incoming E2-ubiquitin conjugates [16].

\section{Substrate}

Whereas both the E2 and the target protein are chemically altered during ubiquitination, for clarity we use the term 'substrate' to refer to the ubiquitinated target and not the E2. The two essential substrates of the APC are securin and the mitotic cyclins (Figure 1) [35]. The degradation of securin triggers sister-chromatid separation, and the degradation of the mitotic cyclins is required for the completion of mitosis. The APC, particularly when bound to Cdh1, also ubiquitinates numerous other proteins involved in various aspects of mitotic exit [5].

Substrates bind specifically to the APC-activator complex through degradation sequences, the best understood of which are the D-box (RXXLXXXN) and KEN-box (KEN) [36,37]. Although D- and KEN-box sequences are required for the ubiquitination of many substrates by the APC, they are often not sufficient, suggesting that substrates contain additional unidentified degradation sequences [36,37]. Numerous APC substrates contain non-canonical degradation sequences that lack any clear sequence similarities [38-45]. It is likely that most, if not all, APC substrates contain multiple degradation sequences and might therefore be capable of multivalent interactions with the APC-activator complex.

Degradation sequences and ubiquitinated lysines are often found in substrate regions that are likely to be disordered. For example, the globular Cdk-binding domain of cyclins is generally preceded by a disordered amino-terminal region that contains the critical D- and KEN-box sequences, along with numerous lysines. Securin is also likely to possess a disordered amino-terminal APC-recognition region adjacent to a carboxy-terminal functional domain. The separation of degradation and functional domains might prevent the 
degradation signal from interfering with the normal function of the protein, and thus might facilitate the evolution of regulatory degradation. As unfolded sequences at the amino or carboxyl termini of proteins are required for their efficient unfolding and translocation into the proteasome pore for degradation [46,47], these unfolded regions may be a hallmark of all degradation targets.

\section{E2}

E2s share a conserved core domain of approximately 150 amino acids, including the central cysteine residue at which ubiquitin is attached; some E2s also contain aminoor carboxy-terminal extensions that lend specificity to their functions. E2s are charged with ubiquitin by the ubiquitinactivating enzyme E1 (Figure 2a). As E2s use the same binding interface to interact with both the E1 and the E3, E2s must dissociate from the $\mathrm{E}_{3}$ to be recharged with ubiquitin [48]. The rate of E2 turnover is very fast: during in vitro ubiquitination experiments, the APC can add as many as ten ubiquitins to a substrate within seconds (MR-B and MEM, unpublished results).

Ubiquitin-protein ligases such as the APC catalyze two distinct reactions: the ligation of ubiquitins to different substrate lysines (termed multiple monoubiquitination) and the transfer of ubiquitins to specific lysines on previously attached ubiquitins, leading to the formation of ubiquitin chains (termed polyubiquitination). Lysine specificity is determined primarily by the E2. In yeast, for example, Ubc4 promotes addition of ubiquitins to substrate lysines, whereas Ubc1 catalyzes ubiquitination of lysine 48 (K48) of a previously attached ubiquitin, leading to K48-linked chains that are recognized by the proteasome [49]. The different preferences of these E2s allow them to collaborate in vivo, such that Ubc4 attaches the initial ubiquitins to substrate lysines and Ubc1 extends these ubiquitins into K48-linked chains. In vertebrates, $\mathrm{UbcH}_{5}$, like its yeast ortholog Ubc4, tends to generate nonspecific linkages to substrate lysines [50,51], whereas E2-25K, like yeast Ubc1, generates K48-linked chains [49,52]. UbcH1O allows the APC to make K11-linked chains [51].

\section{The APC reaction}

We now examine how the components of the APC reaction work together to catalyze the varied ubiquitination activities of the APC.

\section{Substrate binding}

Activators clearly contribute to the recognition of specific substrates for ubiquitination by the APC, but there is evidence that substrates also bind to the APC core: a peptide containing repeated D-boxes binds directly to the APC in the absence of activators [53]. The location of the D-box receptor is not known, but one possibility is the APC subunit Doc1. Doc1 enhances substrate binding to the APCactivator complex [33,34], thereby increasing substrate residence time and increasing the number of ubiquitins that are attached in a single substrate-binding event [54]. The enhanced substrate affinity provided by Doc1 is dependent on the substrate D-box [55].

Notably, Doc1 does not promote substrate binding by stimulating binding of the activator subunit. The concentration of activator required for half-maximal APC stimulation is unaffected by the removal of Doc1 [54]. In addition, steady-state activator binding and the rate of activator dissociation from the APC are unaffected by mutations in Doc1 that are known to reduce substrate binding [34]. Thus, Doc1 seems to promote activatorindependent substrate binding to the core APC.

Several models of substrate recognition by the APC have been proposed to take into account both activator and APC core binding sites [56]. One possibility is a multivalent mechanism, where substrates are shared between substrate receptors on both the activator and APC. Multivalent substrate binding is supported by the observation that substrate enhances the affinity of activator for the APC, through a mechanism that depends on Doc1 $[34,57]$. The simplest explanation for these results is that a multivalent substrate binds simultaneously to both activator and APC, although it is not possible to rule out more complex mechanisms such as substrate-induced conformational changes in the activator. A multivalent substrate-binding model is attractive for several reasons. Multiple lowaffinity interactions would explain why individual interactions between substrates and the APC have been so difficult to detect, and allow high-affinity substrate binding to the APC-activator complex despite low-affinity individual interactions. Multivalency might also allow greater specificity in substrate recognition by requiring two or more separate binding interactions for efficient substrate recognition.

\section{Catalysis}

After substrate binding, ubiquitin transfer requires the nucleophilic attack of the E2-ubiquitin thioester bond by the $\varepsilon$-amino group of a substrate lysine, leading to the formation of an isopeptide bond between the carboxyl terminus of ubiquitin and the substrate lysine by way of an oxyanion intermediate (Figure 5). This reaction depends on the deprotonation of the attacking amide group, as well as stabilization of the negatively charged oxyanion intermediate $[1,58,59]$.

The catalytic residues that promote ubiquitin transfer reside in the active site of the E2 itself. Biochemical studies of Ubc9, which conjugates the ubiquitin-like protein SUMO, have identified residues conserved in most E2S that enhance deprotonation of the substrate lysine - not by abstracting a proton from the lysine but by creating a local microenvironment of reduced lysine $\mathrm{pK}$ [6o]. In addition, 


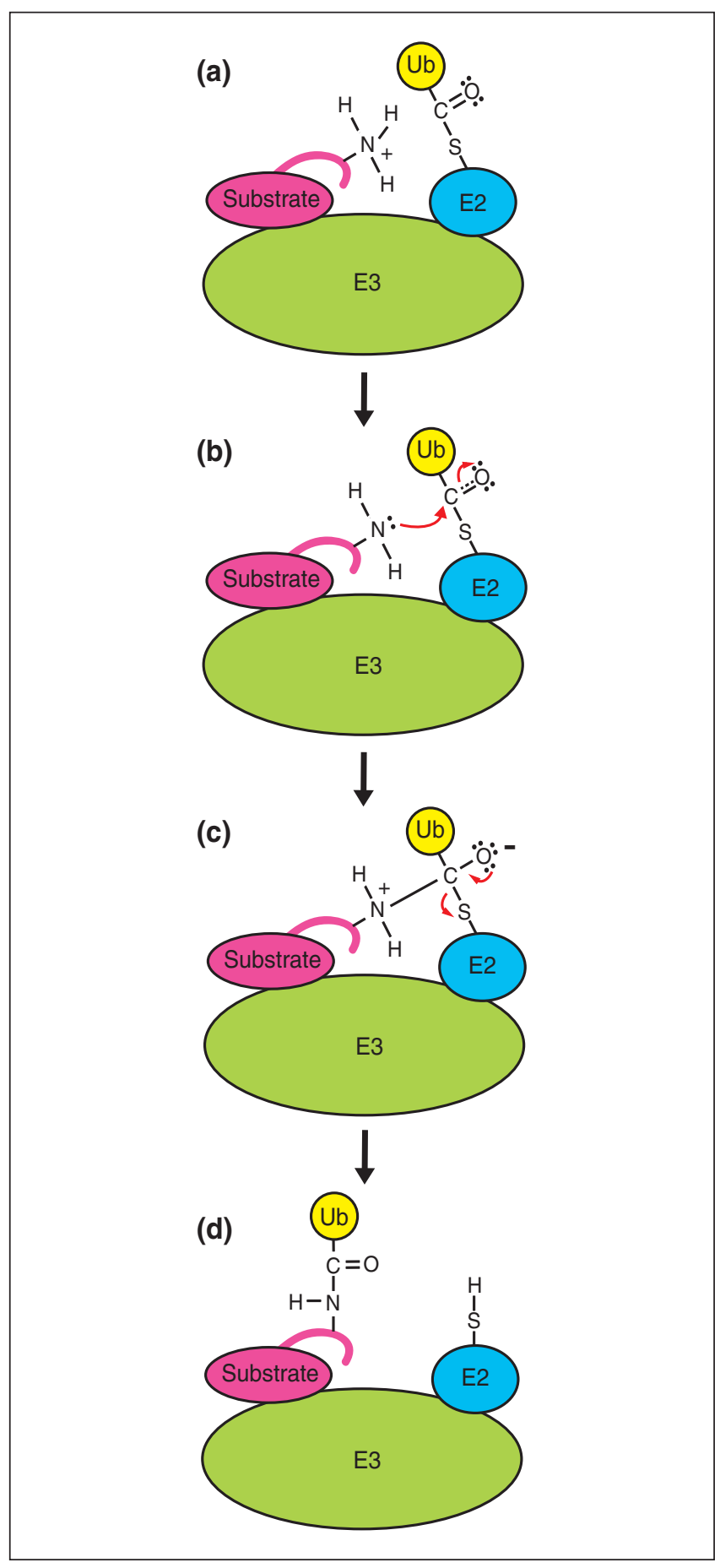

Figure 5

Reaction mechanism underlying protein ubiquitination by the APC and other RING-domain ubiquitin ligases. (a) The E3 positions the substrate and E2-ubiquitin conjugate in close proximity.

(b) Deprotonation of the $\varepsilon$-amino group of a lysine on the substrate promotes nucleophilic attack of the thioester bond between the ubiquitin carboxyl terminus and the E2 active-site cysteine.

(c) The initial product of nucleophilic attack is an oxyanion intermediate. (d) Completion of the reaction results in an isopeptide bond between the ubiquitin carboxyl terminus and the substrate lysine. a conserved asparagine in the E2 might provide a positive charge that stabilizes the oxyanion intermediate [59]. Although this residue is positioned far from the E2 active site in structures of unconjugated E2s, it rotates towards the active-site cysteine upon ubiquitin or SUMO conjugation, or E3 binding [61]. It therefore appears that the ubiquitin-protein ligase does not catalyze the chemical step directly, but instead facilitates ubiquitination by positioning the substrate and E2 in a productive orientation.

Because the catalytic residues for ubiquitin transfer are located within the E2, high concentrations of substrate lysines drive a low rate of ubiquitin transfer from an E2-ubiquitin conjugate, even in the absence of an $\mathrm{E}_{3}[62,63]$ (MR-B and DOM, unpublished results). This activity can be detected with a diubiquitin synthesis assay, which measures transfer of ubiquitin from the E2 to monomeric ubiquitin in solution. At least in some cases (including the E3s SCF and APC), the rate of ubiquitin transfer by E2s is stimulated by interaction with a RING E3 [62,64,65]. Because the ubiquitin substrate in these experiments is free in solution and is therefore not positioned by the $\mathrm{E}_{3}$, stimulation by the E3 may be due to an activating conformational change induced in the $\mathrm{E} 2$ by the RING domain.

Activator proteins might enhance the rate of ubiquitin transfer by the APC through a mechanism that is distinct from their role in substrate binding. Studies of the unusual APC substrate Nek2A suggest that activators induce an activating conformational change in the APC. Nek2A contains a carboxy-terminal dipeptide motif (MR) that is similar to the IR motif at the carboxyl terminus of activators, and it uses this motif to bind the APC in the absence of activator. However, bound Nek2A is not ubiquitinated unless activator is also added [66]. Ubiquitination of Nek2A is even stimulated by an amino-terminal fragment of Cdc20 that lacks the substrate-binding WD40 domain [67]. The amino-terminal activator region, which includes the C-box, might therefore promote an activating change in APC structure that is independent of the activator's substrate-recruiting function.

Insights into the role of activator-induced conformational changes in the APC might be found in recent studies of the ubiquitin-protein ligase, SCF. Modification of the cullin subunit of SCF with the ubiquitin-like protein Nedd8 enhances catalytic rate [63], probably as a result of a conformational change that reduces the distance between the RING domain (where the E2 binds) and the substratebinding site [68]. While there is no evidence that the APC is neddylated, it is possible that activator binding to the APC results in a similar conformational change in the cullin subunit Apc2, thereby enhancing the rate of ubiquitination.

Ubiquitination by the APC is processive; that is, multiple ubiquitins are added during a single substrate-binding 
event. Processivity is readily observed in ubiquitination assays with the E2 Ubc4, in which multiubiquitinated substrates appear in the first seconds of a ubiquitination reaction despite the presence of a large excess of unmodified substrate. In these Ubc4-dependent reactions, the APC does not display higher activity with preubiquitinated substrates, and thus would not preferentially re-bind them. Thus, substrates must remain bound to the APC for a sufficient time to allow multiple E2s to bind, transfer ubiquitin, and dissociate. The residence time of a substrate on the APC is determined by its dissociation rate. This rate varies among different substrates, so that different substrates acquire a different number of ubiquitins in a single binding event. Substrate dissociation rate, and thus the degree of processivity, is influenced by the activator subunit: for example, Cdc2o may have a higher affinity for securin than Cdh1, as this substrate is ubiquitinated with higher processivity by APC-Cdc2O than it is by APC-Cdh1 [34].

An increase in the number of ubiquitins added to a substrate may increase the likelihood of substrate recognition by the proteasome in the face of competing deubiquitinating activities in the cell. Thus, substrates that are ubiquitinated more processively by the APC may have an enhanced degradation rate in vivo [69]. However, this variable degradation efficiency could be achieved by varying substrate affinities whether the reaction is processive or not, and so it is not clear what advantage a processive ubiquitination mechanism might confer.

The lysine to which ubiquitin is attached determines the efficiency of substrate recognition by the proteasome. Polyubiquitin chains linked by $\mathrm{K} 48$ of ubiquitin are generally considered to be the ideal proteasome-targeting signal [70]. However, multiple monoubiquitinations and small nonspecific chains might also be sufficient for proteasomal targeting in some cases [49,50], and recent evidence suggests that linkages at K11 or indeed any lysine other than K63 can be recognized by the proteasome $[51,71]$. In yeast, the APC (with the E2 Ubc1) generates polyubiquitin chains linked by $\mathrm{K} 48$; in human cells, the APC (with UbcH10) produces K11-linked chains [51].

As mentioned earlier, E2s have different lysine preferences. In yeast, for example, Ubc4 is specialized for promoting ubiquitin transfer to lysines on a large number of substrates, while Ubc1 is specialized to promote the formation of K48-linked polyubiquitin. The molecular basis of this lysine specificity is not yet understood, but presumably depends on residues surrounding the activesite cysteine of the E2, which are expected to position the attacking lysine [62,72]. Although Ubc1 has a carboxyterminal extension containing a ubiquitin-binding domain, this domain is not required for the K48 specificity of the Ubc1 catalytic domain [49]; instead, this specificity is

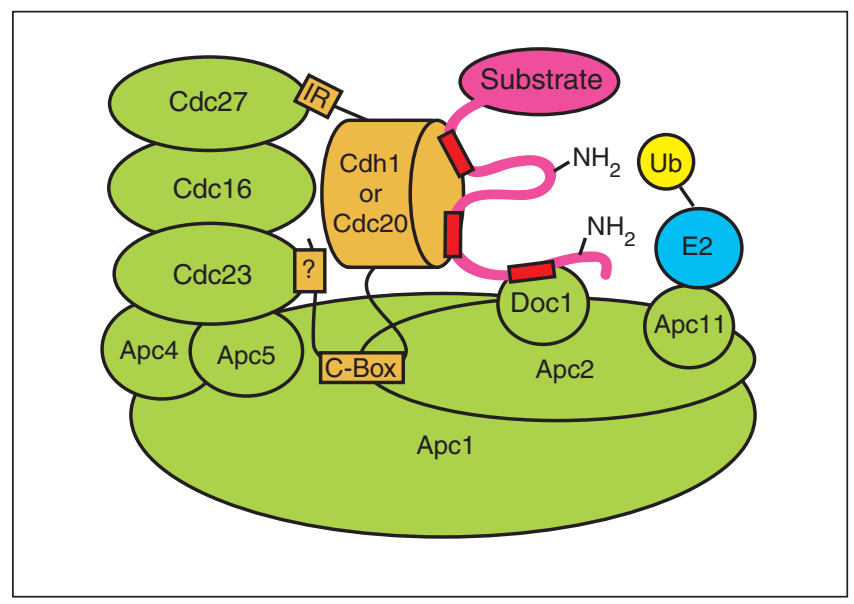

Figure 6

A speculative model of protein ubiquitination by the APC. Substrate (magenta) is likely to bind multivalently to both activator and the APC using degradation sequences (red rectangles) in disordered substrate regions. The APC positions substrates so that multiple lysine side chains can effectively attack the E2-ubiquitin bond. Residues near the E2 active site promote catalysis, which is stimulated upon binding to the APC. Flexibility in both the substrate and APC may help accommodate the modification of many lysines and the formation of long polyubiquitin chains.

provided by specific residues near the active-site cysteine in the catalytic domain (MR-B and DOM, unpublished results).

The ability to modify many different lysines and accommodate growing ubiquitin chains implies some degree of flexibility in the APC active site. As discussed earlier, many APC substrates are predicted to contain unstructured amino-terminal sequences, which would allow variable positioning of attacking lysines and might also allow a growing substrate to loop out of the active site. Multivalent substrate binding might allow the APC to better position these unstructured regions for attack. Furthermore, the APC core may also provide a source of flexibility. EM structural analysis suggests that the angle between the 'arc lamp' and the 'platform' might vary to accommodate substrates of various sizes or ubiquitin chain length [16]. In addition, neddylation of some cullin-RING ligases results in more flexible tethering of the RING domain to the associated cullin subunit, providing flexibility in the positioning of the RING-E2 complex [68]. Perhaps similar conformational changes occur in the APC upon activator binding, as mentioned earlier.

\section{A model for ubiquitination by the APC}

This leaves us with our current view of protein ubiquitination by the APC (Figure 6). First, an activator binds with very high affinity to the APC core, possibly inducing a conformational change that results in more productive 
positioning of the binding sites for the E2 and substrate. A substrate then binds to the APC-activator complex using multiple degradation sequences, often found in disordered regions, that interact with sites on both the activator and APC core, possibly via the Doc1 subunit. The E2-ubiquitin conjugate then binds to the APC, positioning the E2 near the substrate and perhaps resulting in a conformational change in the E2 that stimulates its activity. Residues near the E2 active site then promote catalysis. Several E2s may cycle on and off the APC before one is successfully attacked by a substrate lysine; in yeast, the initial successful attack is likely to be on a ubiquitin conjugated to Ubc4. After this initial ubiquitin is transferred to the substrate and the E2 dissociates, additional E2s will then bind and be attacked successfully by lysines on the substrate or by specific lysines on ubiquitin itself, as determined by residues near the active site of the E2. Flexibility in the substrate and APC helps accommodate the modification of many different lysines and the formation of long ubiquitin chains. Substrate residence time, and thus the number of ubiquitins added in a single substrate-binding event, is determined by substrate dissociation rate.

Many features of this model remain mysterious. Substrate recognition is still poorly understood: while the roles of some APC subunits are becoming clear, a direct binding site for substrates on the APC core has not been identified, and the amino acid sequences that target substrates to the APC-activator complex are still poorly defined. Activators clearly play a central role in APC function, but it is not clear how these proteins alter enzymatic activity: they may do more to activate the APC than just participate in substrate binding, and we need to know more about the conformational changes, if any, that activators induce in the APC core. Finally, it is unclear how E2s are stimulated upon binding to the RING domain, and the structural determinants of E2 lysine specificity remain to be identified. Clearly, an understanding of these and many other problems will be found in a combination of detailed structural studies and quantitative analyses of proteinprotein interactions and enzyme kinetics.

\section{References}

1. Pickart $\mathrm{CM}$, Eddins $\mathrm{MJ}$ : Ubiquitin: structures, functions, mechanisms. Biochim Biophys Acta 2004, 1695:55-72.

2. Kerscher O, Felberbaum R, Hochstrasser M: Modification of proteins by ubiquitin and ubiquitin-like proteins. Annu Rev Cell Dev Biol 2006, 22:159-180.

3. Peters JM: The anaphase promoting complex/cyclosome: a machine designed to destroy. Nat Rev Mol Cell Biol 2006, 7:644-656.

4. Thornton BR, Toczyski DP: Precise destruction: an emerging picture of the APC. Genes Dev 2006, 20:3069-3078.

5. Sullivan M, Morgan DO: Finishing mitosis, one step at a time. Nat Rev Mol Cell Biol 2007, 8:894-903.

6. Morgan DO: The Cell Cycle: Principles of Control. London: New Science Press; 2007.

7. Deshaies RJ, Joazeiro CA: RING domain E3 ubiquitin ligases. Annu Rev Biochem 2009, 78:399-434.
8. Thornton BR, Ng TM, Matyskiela ME, Carroll CW, Morgan DO, Toczyski DP: An architectural map of the anaphase-promoting complex. Genes Dev 2006, 20:449-460.

9. Dube P, Herzog F, Gieffers C, Sander B, Riedel D, Muller SA, Engel A, Peters JM, Stark H: Localization of the coactivator Cdh1 and the cullin subunit Apc2 in a cryo-electron microscopy model of vertebrate APC/C. Mol Cell 2005, 20:867879.

10. Ohi MD, Feoktistova A, Ren L, Yip C, Cheng Y, Chen JS, Yoon HJ, Wall JS, Huang Z, Penczek PA, Gould KL, Walz T: Structural organization of the anaphase-promoting complex bound to the mitotic activator SIp1. Mol Cell 2007, 28:871885.

11. Schwickart $M$, Havlis J, Habermann B, Bogdanova A, Camasses A, Oelschlaegel T, Shevchenko A, Zachariae W: Swm1/Apc13 is an evolutionarily conserved subunit of the anaphase-promoting complex stabilizing the association of Cdc16 and Cdc27. Mol Cell Biol 2004, 24:3562-3576.

12. Zachariae W, Shevchenko A, Andrews PD, Ciosk R, Galova M, Strak MJR, Mann M, Nasmyth K: Mass spectrometric analysis of the anaphase-promoting complex from yeast: identification of a subunit related to cullins. Science 1998, 279: 1216-1219.

13. Wang J, Dye BT, Rajashankar KR, Kurinov I, Schulman BA: Insights into anaphase promoting complex TPR subdomain assembly from a CDC26-APC6 structure. Nat Struct Mol Biol 2009, 16:987-989.

14. Gieffers C, Dube P, Harris JR, Stark H, Peters JM: Threedimensional structure of the anaphase-promoting complex. Mol Cell 2001, 7:907-913.

15. Passmore LA, Booth CR, Venien-Bryan C, Ludtke SJ, Fioretto C, Johnson LN, Chiu W, Barford D: Structural analysis of the anaphase-promoting complex reveals multiple active sites and insights into polyubiquitylation. Mol Cell 2005, 20:855866.

16. Herzog F, Primorac I, Dube P, Lenart P, Sander B, Mechtler K, Stark H, Peters JM: Structure of the anaphase-promoting complex/cyclosome interacting with a mitotic checkpoint complex. Science 2009, 323:1477-1481.

17. Oelschlaegel $T$, Schwickart $M$, Matos J, Bogdanova A, Camasses A, Havlis J, Shevchenko A, Zachariae W: The yeast APC/C subunit Mnd2 prevents premature sister chromatid separation triggered by the meiosis-specific APC/C-Ama1. Cell 2005, 120:773-788.

18. Rudner AD, Hardwick KG, Murray AW: Cdc28 activates exit from mitosis in budding yeast. J Cell Biol 2000, 149:13611376.

19. Rudner AD, Murray AW: Phosphorylation by Cdc28 activates the Cdc20-dependent activity of the anaphase-promoting complex. J Cell Biol 2000, 149:1377-1390.

20. Kramer ER, Scheuringer N, Podtelejnikov AV, Mann M, Peters JM: Mitotic regulation of the APC activator proteins CDC20 and CDH1. Mol Biol Cell 2000, 11:1555-1569.

21. Kraft C, Herzog F, Gieffers C, Mechtler K, Hagting A, Pines J, Peters JM: Mitotic regulation of the human anaphase-promoting complex by phosphorylation. EMBO J 2003, 22: 6598-6609.

22. Shteinberg M, Protopopov $Y$, Listovsky T, Brandeis M, Hershko $A$ : Phosphorylation of the cyclosome is required for its stimulation by Fizzy/cdc20. Biochem Biophys Res Commun 1999, 260:193-198.

23. Golan A, Yudkovsky Y, Hershko A: The cyclin-ubiquitin ligase activity of cyclosome/APC is jointly activated by protein kinases Cdk1-cyclin B and PIk. J Biol Chem 2002, 277: 15552-15557.

24. Zachariae W, Schwab M, Nasmyth K, Seufert W: Control of cyclin ubiquitination by CDK-regulated binding of Hct1 to the Anaphase Promoting Complex. Science 1998, 282:17211724.

25. Jaspersen SL, Charles JF, Morgan DO: Inhibitory phosphorylation of the APC regulator Hct1 is controlled by the kinase Cdc28 and the phosphatase Cdc14. Curr Biol 1999, 9:227-236 
26. Kraft C, Vodermaier HC, Maurer-Stroh $S$, Eisenhaber $F$ Peters JM: The WD40 propeller domain of Cdh1 functions as a destruction box receptor for APC/C substrates. $\mathrm{Mol}$ Cell 2005, 18:543-553.

27. Burton JL, Solomon MJ: D box and KEN box motifs in budding yeast HsI1p are required for APC-mediated degradation and direct binding to Cdc20p and Cdh1p. Genes Dev 2001, 15:2381-2395.

28. Hilioti Z, Chung YS, Mochizuki Y, Hardy CF, Cohen-Fix O: The anaphase inhibitor Pds1 binds to the APC/C-associated protein $\mathrm{Cdc20}$ in a destruction box-dependent manner. Curr Biol 2001, 11:1347-1352.

29. Schwab M, Neutzner M, Mocker D, Seufert W: Yeast Hct1 recognizes the mitotic cyclin $\mathrm{Clb2}$ and other substrates of the ubiquitin ligase APC. EMBO J 2001, 20:5165-5175.

30. Schwab M, Lutum AS, Seufert W: Yeast Hct1 is a regulator of Clb2 cyclin proteolysis. Cell 1997, 90:683-693.

31. Visintin R, Prinz S, Amon A: CDC20 and CDH1: a family of substrate-specific activators of APC-dependent proteolysis. Science 1997, 278:460-463.

32. Vodermaier HC, Gieffers C, Maurer-Stroh S, Eisenhaber F, Peters JM: TPR subunits of the anaphase-promoting complex mediate binding to the activator protein CDH1. Curr Biol 2003, 13:1459-1468.

33. Passmore LA, McCormack EA, Au SW, Paul A, Willison KR, Harper JW, Barford D: Doc1 mediates the activity of the anaphase-promoting complex by contributing to substrate recognition. EMBO $\mathrm{J} 2003,22: 786-796$

34. Matyskiela ME, Morgan DO: Analysis of activator-binding sites on the APC/C supports a cooperative substratebinding mechanism. Mol Cell 2009, 34:68-80.

35. Thornton BR, Toczyski DP: Securin and B-cyclin/CDK are the only essential targets of the APC. Nat Cell Biol 2003, 5: 1090-1094.

36. Glotzer M, Murray AW, Kirschner MW: Cyclin is degraded by the ubiquitin pathway. Nature 1991, 349:132-138.

37. Pfleger CM, Kirschner MW: The KEN box: an APC recognition signal distinct from the $D$ box targeted by Cdh1. Genes Dev 2000, 14:655-665.

38. Araki M, Wharton RP, Tang Z, Yu H, Asano M: Degradation of origin recognition complex large subunit by the anaphase-promoting complex in Drosophila. EMBO J 2003, 22:6115-6126.

39. Benanti JA, Matyskiela ME, Morgan DO, Toczyski DP: Functionally distinct isoforms of Cik1 are differentially regulated by APC/C-mediated proteolysis. Mol Cell 2009, 33:581-590.

40. Castro A, Vigneron S, Bernis C, Labbe JC, Lorca T: Xkid is degraded in a D-box, KEN-box, and A-box-independent pathway. Mol Cell Biol 2003, 23:4126-4138.

41. Enquist-Newman M, Sullivan M, Morgan DO: Modulation of the mitotic regulatory network by APC-dependent destruction of the Cdh1 inhibitor Acm1. Mol Cell 2008, 30:437-446.

42. Hildebrandt ER, Hoyt MA: Cell cycle-dependent degradation of the Saccharomyces cerevisiae spindle motor Cin8p requires $\mathrm{APC}(\mathrm{Cdh} 1)$ and a bipartite destruction sequence. Mol Biol Cell 2001, 12:3402-3416.

43. Ko N, Nishihama R, Tully GH, Ostapenko D, Solomon MJ, Morgan DO, Pringle JR: Identification of yeast IQGAP (Iqg1p) as an anaphase-promoting-complex substrate and its role in actomyosin-ring-independent cytokinesis. Mol Biol Cell 2007, 18:5139-5153.

44. Littlepage LE, Ruderman JV: Identification of a new APC/C recognition domain, the $A$ box, which is required for the Cdh1-dependent destruction of the kinase Aurora-A during mitotic exit. Genes Dev 2002, 16:2274-2285.

45. Sullivan M, Morgan DO: A novel destruction sequence targets the meiotic regulator Spo13 for anaphase-promoting complex-dependent degradation in anaphase I. J Biol Chem 2007, 282:19710-19715.
46. Prakash S, Tian L, Ratliff KS, Lehotzky RE, Matouschek A: An unstructured initiation site is required for efficient proteasome-mediated degradation. Nat Struct $\mathrm{Mol}$ Biol 2004, 11:830-837.

47. Prakash $\mathrm{S}$, Inobe $\mathrm{T}$, Hatch AJ, Matouschek A: Substrate selection by the proteasome during degradation of protein complexes. Nat Chem Biol 2009, 5:29-36.

48. Eletr ZM, Huang DT, Duda DM, Schulman BA, Kuhlman B: E2 conjugating enzymes must disengage from their $E 1$ enzymes before E3-dependent ubiquitin and ubiquitinlike transfer. Nat Struct Mol Biol 2005, 12:933-934.

49. Rodrigo-Brenni M, Morgan DO: Sequential E2s drive polyubiquitin chain assembly on APC targets. Cell 2007, 130:127-139.

50. Kirkpatrick DS, Hathaway NA, Hanna J, Elsasser S, Rush J, Finley D, King RW, Gygi SP: Quantitative analysis of in vitro ubiquitinated cyclin B1 reveals complex chain topology. Nat Cell Biol 2006, 8:700-710.

51. Jin L, Williamson A, Banerjee S, Philipp I, Rape M: Mechanism of ubiquitin-chain formation by the human anaphase-promoting complex. Cell 2008, 133:653-665.

52. Chen Z, Pickart CM: A 25-kilodalton ubiquitin carrier protein (E2) catalyzes multi-ubiquitin chain synthesis via lysine 48 of ubiquitin. J Biol Chem 1990, 265:21835-21842.

53. Yamano H, Gannon J, Mahbubani H, Hunt T: Cell cycle-regulated recognition of the destruction box of cyclin B by the APC/C in Xenopus egg extracts. Mol Cell 2004, 13:137147.

54. Carroll CW, Morgan DO: The Doc1 subunit is a processivity factor for the anaphase-promoting complex. Nat Cell Biol 2002, 4:880-887.

55. Carroll CW, Enquist-Newman M, Morgan DO: The APC subunit Doc1 promotes recognition of the substrate destruction box. Curr Biol 2005, 15:11-18.

56. Yu H: Cdc20: a WD40 activator for a cell cycle degradation machine. Mol Cell 2007, 27:3-16.

57. Burton JL, Tsakraklides V, Solomon MJ: Assembly of an APC-Cdh1-substrate complex is stimulated by engagement of a destruction box. Mol Cell 2005, 18:533-542.

58. Passmore LA, Barford D: Getting into position: the catalytic mechanisms of protein ubiquitylation. Biochem $J$ 2004, 379:513-525.

59. Wu PY, Hanlon M, Eddins M, Tsui C, Rogers RS, Jensen JP, Matunis MJ, Weisman AM, Wolberger C, Pickart CM: A conserved catalytic residue in the ubiquitin-conjugating enzyme family. EMBO J 2003, 22:5241-5250.

60. Yunus AA, Lima CD: Lysine activation and functional analysis of E2-mediated conjugation in the SUMO pathway. Nat Struct Mol Biol 2006, 13:491-499.

61. Reverter D, Lima CD: Insights into E3 ligase activity revealed by a SUMO-RanGAP1-Ubc9-Nup358 complex. Nature 2005, 435:687-692.

62. Petroski MD, Deshaies RJ: Mechanism of lysine 48-linked ubiquitin-chain synthesis by the cullin-RING ubiquitinligase complex SCF-Cdc34. Cell 2005, 123:1107-1120.

63. Saha A, Deshaies RJ: Multimodal activation of the ubiquitin ligase SCF by Nedd8 conjugation. Mol Cell 2008, 32:21 31.

64. Gmachl M, Gieffers C, Podtelejnikov AV, Mann M, Peters JM: The RING-H2 finger protein APC11 and the E2 enzyme UBC4 are sufficient to ubiquitinate substrates of the anaphase-promoting complex. Proc Natl Acad Sci USA 2000, 97:8973-8978.

65. Ozkan E, Yu H, Deisenhofer J: Mechanistic insight into the allosteric activation of a ubiquitin-conjugating enzyme by RING-type ubiquitin ligases. Proc Natl Acad Sci USA 2005, 102:18890-18895.

66. Hayes MJ, Kimata Y, Wattam SL, Lindon C, Mao G, Yamano $\mathrm{H}$, Fry AM: Early mitotic degradation of Nek2A depends on Cdc20-independent interaction with the APC/C. Nat Cell Biol 2006, 8:607-614. 
67. Kimata Y, Baxter JE, Fry AM, Yamano H: A role for the Fizzy/ Cdc20 family of proteins in activation of the APC/C distinct from substrate recruitment. Mol Cell 2008, 32:576583.

68. Duda DM, Borg LA, Scott DC, Hunt HW, Hammel M, Schulman BA: Structural insights into NEDD8 activation of cullinRING ligases: conformational control of conjugation. Cell 2008, 134:995-1006.

69. Rape M, Reddy SK, Kirschner MW: The processivity of multiubiquitination by the APC determines the order of substrate degradation. Cell 2006, 124:89-103.

70. Pickart CM, Fushman D: Polyubiquitin chains: polymeric protein signals. Curr Opin Chem Biol 2004, 8:610-616.
71. Xu P, Duong DM, Seyfried NT, Cheng D, Xie Y, Robert J, Rush $\mathrm{J}$, Hochstrasser M, Finley D, Peng J: Quantitative proteomics reveals the function of unconventional ubiquitin chains in proteasomal degradation. Cell 2009, 137:133-145.

72. Eddins MJ, Carlile CM, Gomez KM, Pickart CM, Wolberger C: Mms2-Ubc13 covalently bound to ubiquitin reveals the structural basis of linkage-specific polyubiquitin chain formation. Nat Struct Mol Biol 2006, 13:915-920.

Published: 26 October 2009

doi:10.1186/jbiol184

(c) 2009 BioMed Central Ltd 